\title{
CONSTRUCCIÓN DE UNA DESCOMPOSICIÓN GENÉTICA: ANÁLISIS TEÓRICO DEL CONCEPTO DIFERENCIAL DE UNA FUNCIÓN EN VARIAS VARIABLES
}

\section{CONSTRUCTION OF A GENETIC DECOMPOSITION: THEORETICAL ANALYSIS OF THE CONCEPT OF DIFFERENTIAL OF A FUNCTION IN SEVERAL VARIABLES}

\author{
Zagalo Enrique Suárez-Aguilar ${ }^{1}$
}

Recibido: febrero 16 de 2015

Aceptado: mayo 13 de 2015

\section{Resumen}

Este artículo presenta un modelo de la cognición y la epistemología, una descomposición genética (DG), del concepto de la diferencial de una función en varias variables (DFVV) del pensamiento matemático avanzado (PMA), en términos de las estructuras mentales de Acción, Proceso, Objeto y Esquema (APOE) y de los mecanismos para la construcción de éstas estructuras por abstracción reflexiva. La DG se constituye en un referente para el diseño de estrategias de enseñanza y como instrumento para dar cuenta del comportamiento observable del sujeto en la comprensión del concepto.

Palabras clave: Comprensión, diferencial, función en varias variables, pensamiento matemático avanzado, descomposición genética, APOE.

\begin{abstract}
This paper presents a model of the epistemology and cognition, a genetic decomposition (DG), of the concept of differential of a function in several variables (DFSV) of the advanced mathematical thinking (AMT), in terms of mental structures of Action, Process, Object and Schema (APOS) and of the mechanisms for building these structures by reflective abstraction. The DG becomes a reference for the design of teaching strategies and as tool to account for the observable behavior of the subject in understanding the concept.
\end{abstract}

Keywords: Understanding, differential, function in several variables, advanced mathematical thinking, genetic decomposition, APOS.

\footnotetext{
1 Licenciado en matemáticas y física, magister en ciencias matemáticas, Universidad Pedagógica y Tecnológica de Colombia, Colombia. E-mail: zagalo.suarez@uptc.edu.co
} 


\section{Introducción}

Este estudio tiene como propósito proponer una descomposición genética (DG) de la diferencial de una función de varias variables (DFVV), la cual describe la estructura del concepto y un camino cognitivo que señala cómo un estudiante podría hacer las construcciones mentales necesarias para alcanzar diferentes niveles de comprensión. La DG es un aporte a la Didáctica de las Matemáticas para la enseñanza y aprendizaje de la DFVV que presenta dificultades para su generalización y aplicación.

Estos problemas se manifiestan al utilizar diferentes sistemas de representación y por la traducción que deben hacer los estudiantes entre estos; además, por la complejidad cognitiva debido a las múltiples interacciones entre los aspectos históricos, teóricos, epistemológicos, pedagógicos y didácticos de otros conceptos relacionados, como se evidencia en investigaciones de infinitesimal (Robinson, 1966), (Ohlsson, 1988); función (García \& LLinares, 1996), (Leinhardt et al., 1990), (Baker et al., 2000), (Trigueros \& Martínez-Planell, 2007); límite (Cornu, 1983), (Davis, 1986), (Cottrill et al., 1996), (Sierra et al, 1999), (Sánchez, 2012); derivada (Azcárate, 1990), (Asiala et al., 1997), (Sánchez-Matamoros et al., 2006), (Gutiérrez, 2012); diferenciabilidad (Artigue, 1986), (Phillips, 1931), (Gómez Fuentez \& Delgado Rubí, 2012) e integral (Aldana, 2011).

Las siguientes son algunas de las problemáticas asociadas a la comprensión del concepto de DFVV:

Al considerar aplicaciones definidas sobre abiertos del espacio $n$-dimensional, $\mathbb{R}^{n}, n>1$, carece de sentido considerar cocientes incrementales de tales aplicaciones y por lo tanto no se puede generalizar el concepto de derivada en esos términos, se hace en términos de la diferencial de una función. Sin embargo, es posible generalizar el concepto de derivada a subespacios de dimensión uno, apareciendo así el concepto de derivada direccional y como caso particular la derivada parcial, (Galindo et al., 2005).
La existencia de funciones en dos o más variables que pueden ser continúas respecto a cada variable separadamente y en cambio ser discontinua como función de dos o más variables, ejemplo la función, $(x, y)=\frac{x y}{x^{2}+y^{2}}$ si $(x, y) \neq(0,0), f(0,0)=0$ es discontinua en el punto $(0,0)$. Por tanto, la existencia de las derivadas parciales de una función en un punto no garantiza la existencia de todas las derivadas direccionales de la función en este punto, (Bartle, 1975, p. 356).

Existen funciones que aunque poseen todas las derivadas direccionales en un punto, sin embargo, no son continuas en el punto y por tanto no son diferenciables allí, es decir, la condición para funciones en una variable que la derivabilidad en un punto implica la continuidad en éste, no se cumple para funciones de varias variables. Por ejemplo para la función $f(x, y)=\frac{x y^{2}}{x^{2}+y^{4}}$ si $x \neq 0, f(0, y)=0$, no es continua en (Apostol, 1988, p. 314).

Funciones que poseen las derivadas parciales en un punto pero no son diferenciables en este, por ejemplo la función $f(x, y)=\frac{x y^{2}}{x^{2}+y^{2}}$ si $(x, y) \neq(0,0), f(0,0)=(0,0), \quad$ es continua y las derivadas parciales existen en $(0,0)$, pero no es diferenciable en este punto, (Bartle, 1975, p. 352).

A pesar de estas dificultades, hay pocas investigaciones sobre la enseñanza y aprendizaje de la DFVV, que es un concepto fundamental del análisis matemático, importante en las ciencias, ingenierías, ciencias sociales y administrativas (Trigueros \& Martínez-Planell, 2007), y su desarrollo es uno de los logros contundentes de la ciencia (Thomas, Finney, \& Weir, 1999).

Un aporte que se presenta para superar el problema descrito anteriormente es estudiar y analizar la comprensión de conceptos matemáticos como objeto de investigación, siguiendo el enfoque teórico y metodológico denominado, Acciones-Procesos-Objetos-Esquema, APOE. Esta teoría, establece un ciclo de investigación que comprende: 
el análisis teórico, el diseño e implementación de instrucción y la recolección y análisis de datos. En este estudio se desarrolla la componente análisis teórico del objeto matemático la diferencial, presentando como resultado la propuesta de una DG la cual orientará el desarrollo de las siguientes fases del ciclo, el diseño e implementación de instrucción y la recolección y análisis de datos, donde se determinará la validez de este primer análisis como resultado de los datos empíricos obtenidos, para obtener un modelo de cognición que permita describir las concepciones de los estudiantes y para diseñar estrategias efectivas de instrucción; éstos resultados se presentarán en un próximo informe.

El enfoque APOE ha sido desarrollado y validado por la comunidad de educación matemática para el estudio de la cognición y la enseñanza de los conceptos, a partir de un análisis de objetos mentales sus transformaciones y relaciones. El enfoque ha demostrado ser pertinente para analizar y dar cuenta de la manera cómo los estudiantes universitarios entienden y son capaces de integrar los conceptos de las matemáticas en un nivel superior, además, los estudios apoyados en la noción de esquema señalan las relaciones en las que hay que hacer mayor énfasis en la docencia y proporcionan indicadores de la forma de hacerlo (Trigueros, 2005, p. 28).

Con lo expuesto se plantea la siguiente pregunta de investigación: ¿Cómo caracterizar los mecanismos y estructuras mentales que promueven la comprensión del concepto DFVV?. La respuesta al anterior interrogante se dará en este artículo con el desarrollo de las siguientes secciones: marco teórico, donde se establecen la definición del concepto de DFVV que se espera que el estudiante logre alcanzar, el enfoque teórico y metodológico APOE, la propuesta de una descomposición genética de la DFVV, las conclusiones y las recomendaciones para estudios posteriores.

\section{Materiales y métodos}

\subsection{Marco teórico}

En esta sección se presenta la definición de DFVV, las características del tipo de pensamiento donde se ubica el concepto, la descripción de las construcciones mentales (acción, proceso, objeto y esquema) y los mecanismos para su construcción por abstracción reflexiva (representar, coordinar, encapsular, generalizar e invertir), que un estudiante necesita realizar para comprender un concepto, presentando ejemplos y situaciones para el objeto de estudio.

Una función $f$ en varias variables, se define como una aplicación sobre un subconjunto $\Omega$ de $\mathbb{R}^{n}$ en $\mathbb{R}^{m}$, con $n \geq 1, m \geq 1$. La función $f$ es diferenciable en un punto interior $a$ de $\Omega$ si existe una transformación lineal $T_{\alpha}$ definida de $\mathbb{R}^{n}$ en $\mathbb{R}^{m}, T_{a}: \mathbb{R}^{n} \rightarrow \mathbb{R}^{m}$,y una función en varias variables $E_{\alpha}$ representada en (1),

$$
\begin{aligned}
E_{a}: \Omega \subseteq \mathbb{R}^{n} & \rightarrow \mathbb{R}^{m} \\
v & \rightarrow E_{a}(v)=E(a, v),
\end{aligned}
$$

tal que,

$$
f(a+v)=f(a)+T_{a}(v)+\|v\| E(a, v) .
$$

Donde $E(a, v) \rightarrow 0$ cuando $v \rightarrow 0$. A la transformación $T_{\alpha}$, se llama la diferencial de en $\boldsymbol{f}$ en $\boldsymbol{\alpha}$ y se nota como $D f(\alpha)$ (Apostol, 1988 p. 329).

Esta definición es equivalente a afirmar que la función $f$ en varias variables, es diferenciable en un punto interior $\boldsymbol{a}$ de $\Omega$ si existe una transformación lineal, $T_{a}: \mathbb{R}^{n} \rightarrow \mathbb{R}^{m}$, tal que el limite cuando $x$ se aproxima a $a$, del cociente entre la norma en $\mathbb{R}^{m}$ del vector, $f(x)-f(a)-T_{a}(x-a)$ y la norma en $\mathbb{R}^{n}$ del vector $x$ - $a$ es cero, ver (3) (Bartle, 1975 p. 349).

$$
\lim _{x \rightarrow a} \frac{\left\|f(x)-f(a)-T_{a}(x-a)\right\|_{m}}{\|x-a\|_{n}}=0
$$


El concepto DFVV, se ubica en el pensamiento matemático avanzado, PMA, que según Tall (1991) es un fenómeno en el que interactúan los procesos mentales para representar, visualizar, generalizar, clasificar, conjeturar, inducir, analizar, sintetizar, abstraer, definir, formalizar y demostrar. Además, se caracteriza por la complejidad de los contenidos y la forma de controlarla, por el rigor y el formalismo que con frecuencia sigue la secuencia teorema, demostración y aplicación. Este tipo de pensamiento es característico en los últimos años de bachillerato y de las matemáticas superiores de la universidad.

Potenciar el PMA, es de interés para la Didáctica de Matemática, a través de la comprensión de los conceptos matemáticos. Un "marco teórico y metodológico para describir cómo los conceptos matemáticos pueden ser comprendidos"es APOE denominada así por sus siglas de, Acción-Proceso-Objeto-Esquema (Arnon et al., 2014, p. 17). Esta teoría establece que el fenómeno de "la comprensión inicia con la manipulación de los objetos físicos o mentales previamente construidos para formar acciones; las acciones se interiorizan para formar procesos, los cuales se encapsulan para formar objetos. Los objetos se pueden volver a desencapsular hacia el proceso desde el cual se formaron. Finalmente las acciones, los procesos y los objetos se organizan en esquemas" (Asiala, 1996, p. 9). El progreso de éste fenómeno se produce cuando la persona reconstruye una situación problemática similar a una ya resuelta pero con un nivel superior de dificultad, (Asiala, 1996).

La teoría APOE reinterpreta el constructivismo a partir de la adaptación de algunas ideas del enfoque cognitivo de Piaget al PMA. Una de estas ideas es la abstracción reflexiva que Piaget la define como "el mecanismo por el cual el individuo se mueve de un nivel de pensamiento a otro de nivel superior y se lleva a cabo a través de la realización de actividades (físicas y mentales) por el sujeto, que tienen dos partes necesariamente asociadas: una es una proyección a un plano superior de pensamiento y la otra es la organización y reconstrucción de aquel conocimiento para formar nuevas estructuras que son estudiadas desde el enfoque cognitivo" (Piaget \& García, 1983, p. 10). Además, considera que este tipo de abstracción es un método por el cual todas las estructuras lógico matemáticas se derivan y conducen a una clase de pensamiento matemático, donde forma o proceso se separan del contenido y los procesos mismos se convierten en objetos de contenido, en la mente del matemático (Piaget et al., 1986).

En la teoría APOE, con el propósito de desarrollar la noción de abstracción reflexiva en el PMA, se aíslan las características esenciales de esta abstracción y se hace un análisis del papel que desempeñan en las matemáticas superiores para formar una teoría de la evolución de los conceptos en este pensamiento que sea coherente de conocimiento y construcción (Dubinsky, 2000). La figura 1, muestra la relación entre los tipos de abstracciones reflexivas o mecanismos mentales de interiorizar, coordinar, encapsular, generalizar, invertir, necesarios para construir estructuras mentales de acciones, procesos, objetos y esquemas; que se definen a continuación (Dubinnsky,1991; tomado de Arnon, Cottrill, Dubinsky, Oktac, Roa Fuentes, Trigueros, Weller, \& Kirk 2014).

Esquema

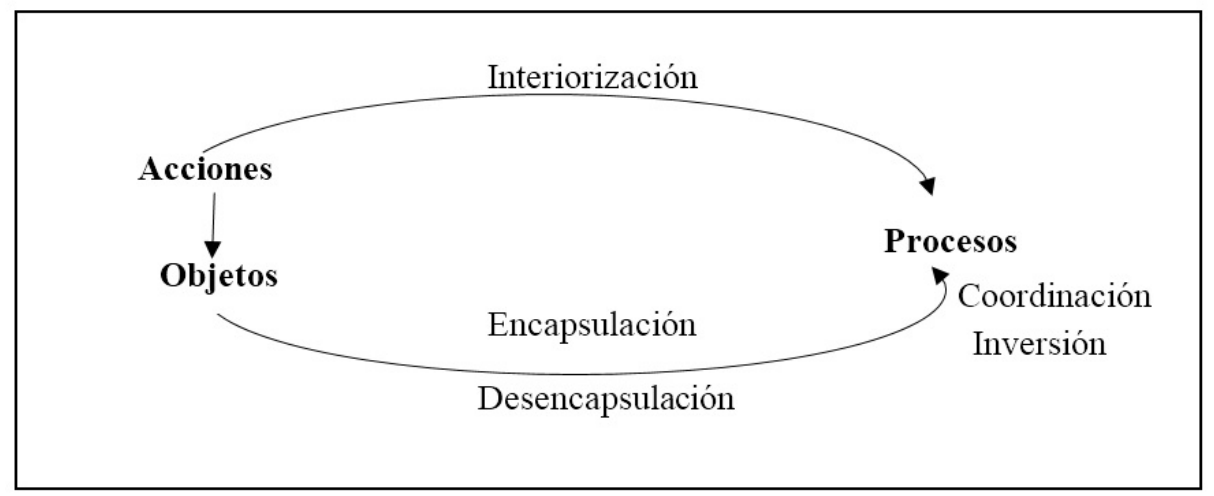

Figura 1. Estructuras y mecanismos mentales de construcción de un concepto. Fuente (Arnon et al., 2014, p.18). 
Representar. Es la capacidad de utilizar símbolos, lenguajes, imágenes, e imágenes mentales, para construir procesos internos como formas de encontrar significados de los fenómenos percibidos. Piaget denominó a esta capacidad como interiorización y se refirió como "la traducción de una serie de acciones materiales en un sistema de operaciones" (Piaget, 1980, p. 90). "La interiorización permite al sujeto ser consciente de una acción, reflexionar sobre esta y combinarla con otras acciones" (Dubinsky, 1991, p. 107).

Coordinar. "Es la composición de dos o más procesos para la construcción de una nueva acción, proceso u objeto" (Tall, 1991, p. 101).

Encapsular. Es la conversión de un proceso (dinámico) en un objeto (estático). "La encapsulación ocurre cuando el individuo reflexiona sobre las operaciones aplicadas a un proceso particular, toma conciencia de éste como un todo y es capaz de realizar y crear transformaciones, sobre éstas acciones o procesos" (Tall, 1991, p. 101).

Generalizar. "Es la capacidad de aplicar un esquema existente particular a un conjunto más amplio de fenómenos y se considera como la forma más simple y más familiar de abstracción reflexiva. La generalización puede ocurrir cuando el sujeto se da cuenta de la aplicabilidad más amplia del esquema o cuando un proceso se encapsula en un objeto" (Tall, 1991, p. 101).

Invertir. "Una vez que un proceso existe internamente es posible para el sujeto invertirlo, no en el sentido de deshacer, sino como un medio de construir un nuevo proceso" (Tall, 1991, p.102).

Se conjetura que la construcción de la mayoría de los conceptos matemáticos se pueden describir en términos de las cinco formas de abstracción reflexiva: interiorización, coordinación, encapsulación, generalización, y la inversión, como lo afirmó Piaget: “Las matemáticas por lo tanto, pueden considerarse en términos de la construcción de estructuras, entidades matemáticas que pasan de un nivel a otro, una operación de tales entidades se convierte a su vez en objeto de la teoría, y este proceso se repite hasta llegar a estructuras que se van alternando para convertirse en estructuras más fuertes" (Piaget, 1972, p. 70).

Con respecto a la estructura mental acción, ésta se considera como "la trasformación o manipulación de objetos que el individuo los percibe como externos. La acción ocurre cuando el sujeto reacciona ante estímulos externos con datos precisos de cómo actuar en cada paso que se da, algunas acciones van más allá del cálculo numérico" (Arnon et al., 2014, p. 19). Por ejemplo ante una situación que involucre la diferencial de un campo escalar $f$, un sujeto con la concepción de acción, requiere conocer la fórmula específica del campo escalar $f$, el dominio $\Omega$ sobre el cual $f$ está definido, el punto interior $a$ de $\Omega$, calcular todas las derivadas parciales de $f$ respecto a cada variable $x$ en $a$ y formar el vector definido en (4), aplicar la transformación y verificar que es lineal, $T_{a}=\nabla f(a)$, evaluarla en algunos vectores específicos $u$ de $\mathbb{R}^{n}$ según (5) y comprobar que satisface (2).

$$
\begin{gathered}
\nabla f(a)=\left(\frac{\partial f(a)}{\partial x_{1}}, \cdots, \frac{\partial f(a)}{\partial x_{n}}\right) . \\
T_{a}: \mathbb{R}^{n} \rightarrow \mathbb{R} \\
u \rightarrow T_{a}(u)=D f(a) \cdot u=\nabla f(a) \cdot u=\left(\frac{\partial f(a)}{\partial x_{1}}, \cdots, \frac{\partial f(a)}{\partial x_{n}}\right) \cdot\left(u_{1}, \cdots, u_{n}\right) .
\end{gathered}
$$


En este caso, las acciones están determinadas por el seguimiento de las instrucciones anteriores y por la notación de los objetos involucrados, pero sin reflexionar sobre los elementos involucrados.

El reflexionar sobre los resultados de la repetición de acciones lleva a la interiorización de las acciones, o por la coordinación entre otros procesos se genera un proceso, que se considera como "una estructura mental que realiza la misma operación que la acción pero ahora totalmente en la mente de la persona, lo que le permite imaginar realizar la transformación sin tener que ejecutar cada paso de forma explícita" (Dubinsky et al., 2005, p. 335; citado por Arnon, et al., 2014).

Por ejemplo, cuando un sujeto repite las acciones anteriores para diferentes campos escalares $f$, cambiando la dimensión del dominio, el punto interior del dominio, él debe reflexionar sobre estas acciones y percibir como aplicaciones dinámicas los campos escalares $f$, los gradientes los campos escalares evaluadas en puntos $\nabla f(a)$, las transformaciones lineales dadas por (5); comenzando así a activar el mecanismo de interiorización que dará origen a una concepción proceso. El sujeto percibe en su mente la diferencial de una función $f$ en un punto a como un proceso, una transformación lineal dada por (5), la cual aplica a cada vector $u$ de $R^{n}$ en el producto punto de $u$ con el vector gradiente de $f$ evaluado en $a, T_{a}(u)=\nabla f(a) \cdot u$, y además que esta transformación junto con la función dada por (1) satisfacen la ecuación (2).

Un sujeto demuestra haber construido una estructura proceso de la DFVV, por ejemplo cuando requiera aplicar la regla de la cadena para diferenciales de campos escalares o vectoriales, el sujeto deberá coordinar dos procesos para componer dos diferenciales.

Por otra parte, un sujeto con la concepción proceso puede invertir los pasos, así, para el caso del campo escalar definido en (6), ante la pregunta, ¿el campo escalar $f$ es diferenciable en el punto $a=(0,0)$ ? . $f: \mathbb{R}^{2} \rightarrow \mathbb{R}$

$(x, y) \rightarrow f(x, y)=2 x+4 y+y^{3}(\sqrt{1-\cos x}+y)$.

Un estudiante que demuestre una concepción proceso podrá identificar que el campo $f$ esta expresado en la forma (2) y podrá invertir los pasos del proceso e inferir que: $f(0,0)=0$, la diferencial de $f$ en $(0,0)$ es el vector $(2,4)$ y que $\|(x, y)\| E_{(0,0)}(x, y)=y^{3}(\sqrt{1-\cos x}+y)$ satisface $E_{(0,0)}(x, y) \rightarrow 0$ cuando $(x, y) \rightarrow(0,0)$.

Otra estructura mental que se considera importante para la comprensión de los conceptos matemáticos, pero la más difícil de lograr, es el objeto. Esta construcción ocurre "cuando un sujeto aplica una acción a un proceso, esto es, demostrar la capacidad de ver la estructura dinámica (proceso) como una estructura estática a la cual se le pueden aplicar acciones" (Arnon et al., 2014, p 22). Al respecto Dubisnky et al (2005) ofrece la siguiente explicación:

"Si una persona se da cuenta del proceso como una totalidad, que las transformaciones pueden actuar sobre esta totalidad y puede realizar tales transformaciones (explícitamente o en la imaginación), entonces decimos que el individuo ha encapsulado el proceso en un objeto cognitivo" ( $p$. 339).

Para el concepto de DFVV, una persona con una concepción proceso debe encapsularlo en objeto o desencapsular el objeto al proceso, para tener éxito ante las siguientes situaciones:

Realizar operaciones con elementos del espacio vectorial de las transformaciones lineales. Dada una función $f$ en varias variables, para definir y realizar operaciones de suma de diferenciales y del producto de la diferencial por un escalar entre elementos del espacio vectorial de las transformaciones lineales de en $\mathbb{R}^{n}$ en $\mathbb{R}^{m}$ según (7), necesita una concepción de objeto de la diferencial de $f$ en el punto o $x, L_{x}=D f(x)$, como un vector de $\mathcal{L}\left(\mathbb{R}^{n}, \mathbb{R}^{m}\right.$. 


$$
\mathcal{L}\left(\mathbb{R}^{n}, \mathbb{R}^{m}\right)=\left\{T: \mathbb{R}^{n} \rightarrow \mathbb{R}^{m}, T(\alpha x+\beta y)=\alpha T(x)+\beta T(y), \alpha \in \mathbb{R}, \beta \in \mathbb{R}, x \in \mathbb{R}^{n}, y \in \mathbb{R}^{n}\right\}
$$

Calcular la norma de un diferencial. Con una concepción objeto de la diferencial de $f$ en $a, D f(a)$, para estimar su norma según (8), la persona requiere desencapsular el objeto, interpretar el vector $D f(a)$ como proceso que trasforma linealmente cada vector $x$ de $\mathbb{R}^{n}$ en un vector $y=D f(a)(x)$ de $\mathbb{R}^{m}$ , y encontrar el supremo del conjunto formado por las normas de los vectores transformados, $\|D f(a)(x)\|_{m} \operatorname{con}\|x\|_{n} \leq 1$.

$$
\|D f(a)\|_{n m}=\sup \left\{\|D f(a)(x)\|_{m}: x \in \mathbb{R}^{n},\|x\|_{n} \leq 1\right\}
$$

Determinar si una función es de clase $C^{1}(\Omega)$. Para establecer si la función $f$ en varias variables es de clase $C^{1}(\Omega)$, es indispensable encapsular el proceso de la diferencial en el objeto $D f(x)$ para todo $x$ en $\Omega$ (interpretar la diferencial como una totalidad, como objetos o vectores de $\mathcal{L}\left(\mathbb{R}^{m}, \mathbb{R}^{n}\right)$ y verificar si la aplicación (9), que a cada vector $x$ le asigna el vector transformación lineal $D f(x)$, es continua. Además, para determinar la continuidad necesita desencapsular la aplicación $D f$ según (7), interpretando cómo actúa la diferencial, para utilizar la medida generada por la norma de la diferencial.

$$
\begin{aligned}
D f: \Omega & \rightarrow \mathcal{L}\left(\mathbb{R}^{m}, \mathbb{R}^{n}\right) \\
x & \rightarrow D f(x) .
\end{aligned}
$$

Una vez construidas las estructuras mentales acciones, procesos y objetos, estas se pueden relacionar y hacer parte de una estructura mental general denominada esquema, para formar un conocimiento individual de un concepto matemático. Además, un esquema elaborado previamente puede ser parte de otro esquema. La formación de esquemas es una actividad dinámica y es un sistema retroalimentado circular. El sujeto tiende a invocar un esquema para entender, tratar, organizar o dar sentido a una situación problema (Arnon et al., 2014).

Por ejemplo, el esquema de diferencial se obtendrá cuando la persona pueda extender la propiedad de aproximar una función diferenciable mediante una función lineal al caso de un número cualquiera de dimensiones y aplicar teoremas de condiciones necesarias y suficientes para la existencia de la diferencial.

Caracterizadas las construcciones mentales, los mecanismos mentales y sus relaciones según el enfoque $A P O E$, estas se pueden representar en un modelo denominado descomposición genética de un concepto, DG. En este modelo se describen los elementos matemáticos que configura el concepto y presenta una trayectoria hipotética de aprendizaje para conjeturar cómo se produce la comprensión aislando pequeñas porciones de las estructuras complejas de pensamiento y dando descripciones explícitas de las posibles relaciones entre las acciones, procesos, objetos y esquemas, (Arnon et al., 2014, p. 28). La DG es una propuesta, producto de observaciones de aprendizaje que el sujeto hace conforme aprende el concepto matemático, que se constituirá en un referente para el diseño de tratamientos de instrucción, además ésta no es única para un concepto (Trigueros, 2005 p.8).

\subsection{Metodología}

Para el diseño de la DG del concepto, se tomó como referencia el ciclo de investigación propuesto por la teoría APOE compuesto por: análisis teórico, diseño e implementación de un tratamiento instruccional y recogida y análisis de información, que se representa en la figura 2, donde las flechas significan que los tres componentes del ciclo de investigación tienen influencia mutua. 


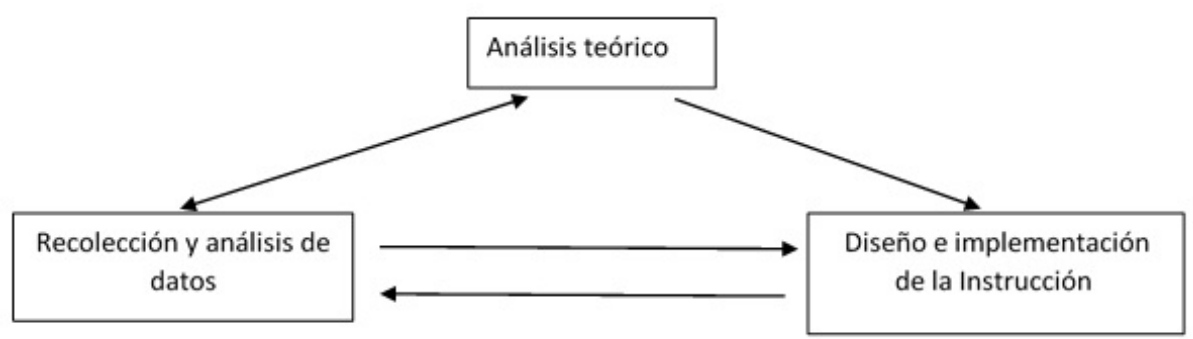

Figura 2. Ciclo de investigación (Arnon et al., 2014)

En este informe se presenta el resultado del análisis teórico, el cual dirige el diseño de actividades, las clases y los ejercicios que al implementar en secuencias de instrucción tienen el propósito de activar los mecanismos mentales para fomentar las construcciones mentales descritas por la DG. La implementación de la instrucción dará oportunidad para la recogida de datos y su análisis que está dirigido por la DG, permitirá responder las siguientes preguntas y el resultado se presentará en un próximo informe: (1) ¿Los estudiantes han construido las estructuras mentales descritas por la DG?, (2) ¿Los estudiantes comprenden correctamente el concepto matemático? Si la respuesta a la primera pregunta es negativa se debe revisar y reconsiderar la instrucción. Si la respuesta a la primera pregunta es afirmativa, sin embargo, la respuesta a la segunda pregunta es negativa se debe revisar y reconsiderar el análisis teórico.

El ciclo se repite hasta cuando las dos respuestas sean afirmativas, en cuyo caso se puede inferir que la evidencia empírica y el análisis teórico describen las mismas construcciones mentales requeridas para comprender el concepto matemático por los estudiantes (Arnon et al., 2014). A continuación se describen los preliminares para el diseño de la DG de la DFVV.

Análisis previo. Se realizó un estudio de investigaciones relacionadas con los esquemas de límite (Cottrill et al., 1996), función (Dubinsky, 2000), derivada (Sánchez-Matamoros et al., 2006), funciones en dos variables (Martínez-Planell \& Trigueros, 2009), espacio vectorial (Arnon et al., 2014), trans- formación lineal (Roa-Fuentes \& Oktac, 2012), concepciones de los estudiantes del concepto de diferencial en matemática y física (Artigue, 1986). Este estudio permitió determinar las concepciones de objeto, proceso o acción de los conceptos previos necesarios para diseñar la DG de la DFVV.

Observaciones y experiencia del investigador. Al desarrollar el concepto de DFVV en cursos de cálculo multivariable en programas de pregrado en ingeniería de la Universidad Pedagógica y Tecnológica de Colombia, UPTC, donde se hace énfasis en la definición, ejercicios y aplicaciones utilizando sistemas de representación gráfico y analítico; y en los programas de matemática y licenciatura en matemática de la UPTC, donde se hace énfasis en la formalización de los conceptos, caracterizado por seguir: definición, teorema, demostración y ejercicios; se determinaron los objetos mentales, las acciones sobre estos objetos, las actividades que promueven alcanzar las concepciones de acción, proceso y de objeto en los estudiantes para caracterizar el esquema del concepto DFVV y diseñar una DG.

Análisis de libros de texto. Se analizó la DFVV e los textos (Thomas, Finney, \& Weir, 1999), (Stewart, 2002), (Galindo, Sanz, \& Tristán, 2005), (Bartle, 1977) y (Apóstol, 1988) y como en esta investigación se optó priorizar el sistema de representación analítico, la definición que se espera que los estudiantes logren de acuerdo a lo establecido por (Trigueros, 2005), es la expuesta en los tres últimos textos analizados y que se presentó en el marco teórico. 


\section{Resultados y discusión}

En este apartado se presenta el procedimiento seguido para resolver las siguientes preguntas de la investigación ¿Qué elementos previos debe poseer el estudiante para alcanzar mayores niveles de comprensión del concepto DFVV?, ¿Cuáles son las estructuras mentales y los mecanismos mentales para su construcción, necesarios para comprender el concepto?.

\subsection{Descomposición genética de la DFVV}

A continuación se describe la descomposición genética del concepto en forma lineal, sin embargo, la comprensión del concepto no es lineal y es posible que se presenten diferentes trayectorias de aprendizaje que incluyan, varios puntos de inicio, saltos o discontinuidades. Además, APOE no descarta la posibilidad que una vez construidas las estructuras mentales estás no aparezcan cuando un sujeto requiera resolver situaciones problemas relacionados (Arnon et al., 2014, p. 40). Estas situaciones se podrán corroborar en las siguientes fases de la investigación del ciclo de investigación.

Construcciones previas. Esquema de función para asimilar el dominio y recorrido como subconjuntos de $\mathbb{R}^{n}, n \geq 1$. El objeto función $f$ en varias variables simbolizado en (8), se desencapsula del proceso del cual se generó para los siguientes casos: Cuando $n=1$ y $m=1$, como una función real de variable real tal que a cada número real del dominio le asigna un único número real; para $n=1$ y $m>1$, función vectorial de una variable real, tal que a cada real del dominio le asigna un único vector en $\mathbb{R}^{m}$; para $n>1$ y $m=1$, función real de una variable vectorial o campo escalar, tal que a cada vector de, le asigna un único real, y para 1 y $m>1$ función vectorial de una variable vectorial o campo vectorial tal que a cada vector en $\mathbb{R}^{n}$ le asigna un único vector en $\mathbb{R}^{m}$.

$$
\begin{aligned}
f: \Omega \subseteq \mathbb{R}^{n} & \rightarrow \mathbb{R}^{m} \\
x & \rightarrow y=f(x) .
\end{aligned}
$$

El esquema de espacio vectorial, formado por la coordinación de los esquemas de conjunto, operación binaria y axioma, que incluyan los objetos base canónica, asimilen el espacio vectorial $\mathbb{R}^{n}, n \geq 1$, sobre el campo $\mathbb{R}$, el producto interior entre dos vectores del mismo espacio, considerar un vector como un objeto para calcular la norma euclídea; el esquema de transformación lineal para asimilar el espacio de las transformaciones lineales sobre el campo $\mathbb{R}$, una concepción objeto de transformación para calcular la norma del supremo.

El esquema de límite de una función de variable real y su generalización a funciones de varias variables.

El esquema de derivada para asimilar el objeto derivada de una función en un punto y la función derivada.

Elementos matemáticos. Desde el enfoque teórico APOE las colecciones de acciones, procesos $u$ objetos son formas de comprender los elementos matemáticos que configuran un concepto (Aldana, 2011, p. 100). Para la DFVV se establecieron los siguientes elementos matemáticos que la configuran: derivada direccional, DD; derivada parcial, DP; derivada de una función de variable real, DEFR; derivada de una función vectorial de variable real, DEFV; derivada de un campo escalar, DECE; derivada de un campo vectorial, DECV y diferencial de una función en varias variables DFVV.

\section{Construcciones a realizar}

\section{A. Derivada de un campo escalar respecto a un vector, $D E$.}

1. Dado los objetos matemáticos, un subconjunto $\Omega$ de $\mathbb{R}^{n}$, la fórmula de un campo escalar particular, $f: \Omega \subseteq \mathbb{R}^{n} \rightarrow \mathbb{R}$, un punto interior $a$ de $\Omega$ y un vector $w$ de $\mathbb{R}^{n}$, realizar las siguientes acciones para calcular la derivada de $f$ en $a$ en dirección del vector $w$. 
a. Definir el campo escalar $F$, como el cociente de diferencias (11), que corresponde al promedio de variación de $f$ en el segmento de recta que une $a$ con $a+h w, h$ un número real. El numerador representa el cambio de $f$ cuando nos desplazamos $a$, hasta $a+h w$.

$$
F(h)=\frac{f(a+h w)-f(a)}{h}
$$

b. Determinar el comportamiento del campo escalar $F$, cuando $h \rightarrow 0$, verificando la existencia del $\lim _{h \rightarrow 0} F(h)$.

c. Si el límite existe en $A 1 b$, denominar a este límite la derivada del campo escalar en el punto en la dirección del vector

2. Interiorizar las acciones en procesos.

a. Repetir las acciones $A 1 a, A 1 b$ y $A 1 c$ para diferentes valores de $a$ y $w$.

b. Repetir A2a para varios campos escalares $f$, cambiando la dimensión $n$ del espacio $\mathbb{R}^{n}$. c. Al realizar $A 2 a, A 2 b$, el mecanismo de interiorización se activa al interpretar derivada de un campo $f$, en un punto $a$ del dominio, en dirección $w$, como una transformación dinámica de estos elementos en número real.

d. Considerar la derivada de cualquier campo $f$ en un punto arbitrario $a$ del dominio, en dirección de vector arbitrario $w$ de $\mathbb{R}^{n}$, como un proceso que transforma estos elementos en un único real dado por, $L=\lim _{h \rightarrow 0} F(h)=\frac{f(a+h w)-f(a)}{h}$, si este existe.

3. Encapsular el proceso $A 2 d$ en un objeto. Considerar el proceso $A 2 d$ como un elemento del conjunto campos escalares $f$, derivables en el punto $\boldsymbol{a}$ del dominio, en dirección de vector $w$ de $\mathbb{R}^{n} \mathrm{y}$ notarlo como $f^{\prime}(a ; w)$.

\section{B. Derivada direccional, DD.}

1. Encapsular el proceso A2d, en el objeto derivada direccional del campo escalar $f$ en la dirección del vector $u$. Interpretar el proceso A2d como un elemento del conjunto de campos escalares derivables en el punto $a$ del dominio, en dirección del vector unitario $u$ de $\mathbb{R}^{n}$ y notarlo como $\mathrm{D}_{u} f(a)$, ver (12).

$$
\mathrm{D}_{u} f(a)=f^{\prime}(a ; u)=\lim _{h \rightarrow 0} \frac{f(a+h u)-f(a)}{h}, \quad \text { si }\|u\|=1 \text {. }
$$

\section{Derivada parcial, DP.}

1. Encapsular el proceso $A 2 d$, en el objeto derivada parcial de $f$ en $a$, respecto a la variable $x_{j}$. Interpretar el proceso $A 2 d$ como un elemento del conjunto de campos escalares derivables en el punto $a$ del dominio en dirección del vector $e_{j}$, (j-ésimo vector coordenado unitario) de $\mathbb{R}^{n}$ y notarlo como $\frac{\partial f}{\partial x_{j}}(a)$, ver (13). Esta concepción se requiere para situaciones como determinar la continuidad de la función $\frac{\partial f}{\partial x_{j}}$ en un punto.

$$
\frac{\partial f}{\partial x_{j}}(a)=\lim _{h \rightarrow 0} \frac{f\left(a+h e_{j}\right)-f(a)}{h}, e_{j}=(0, \cdots, 1, \cdots 0), j=1,2, \cdots, n .
$$




\section{Gradiente de un campo escalar, GCE.}

1. Dados los objetos, un subconjunto de $\Omega$ de $\mathbb{R}^{n}$, la fórmula de un campo escalar, $f: \Omega \subseteq \mathbb{R}^{n} \rightarrow \mathbb{R}$, un punto interior de $\boldsymbol{a}$ de $\Omega$, realizar las siguientes acciones sobre los objetos para calcular el vector gradiente del campo escalar $f$ en $a$.

a. Calcular, si existen, todas las derivadas parciales, $\frac{\partial f}{\partial x_{j}}(a), j=1,2, \cdots, n$.

b. Formar el vector en $\mathbb{R}^{n}$ cuyas componentes son las derivadas parciales calculadas en D1a y denominarlo el vector gradiente de $f$ en el punto $a$,

$$
\left(\frac{\partial f}{\partial x_{1}}(a), \cdots, \frac{\partial f}{\partial x_{n}}(a)\right) \text {. }
$$

2. Interiorizar las acciones en procesos.

a. Realizar las acciones $D 1 a, D 1 b$, para varios valores de.

b. Realizar D2a para varios campos escalares $f$.

c. Coordinar $D 2 a$ y $D 2 b$ en un proceso tal que dado un escalar definido como $f: \Omega \subseteq \mathbb{R}^{n} \rightarrow \mathbb{R}$ , y un punto interior de $a$ de $\Omega$, genera el vector $\left(\frac{\partial f}{\partial x_{1}}(a), \cdots, \frac{\partial f}{\partial x_{n}}(a)\right)$.

3. Encapsular el proceso D2c en el objeto gradiente de un campo escalar definido por (14). Interpretar $\nabla f(a)$ como un objeto sobre el cual se pueden realizar acciones como calcular su norma, $\|\nabla f(a)\|$, caracterizar el objeto $f^{\prime}(a ; u)$ como la componente del objeto $\nabla f(a)$ a lo largo del vector $\boldsymbol{u}$, la cual alcanza el valor máximo cuando la dirección del vector $\nabla f(a)$ coincide con la de $\boldsymbol{u}$.

$$
\nabla f(a)=\left(\frac{\partial f(a)}{\partial x_{1}}, \cdots, \frac{\partial f(a)}{\partial x_{n}}\right) .
$$

\section{E. Derivada de una función real de variable real, DEFR.}

1. Dados los objetos, un subconjunto $\Omega$ de $\mathbb{R}$, la fórmula de una función, $f: \Omega \subseteq \mathbb{R} \rightarrow \mathbb{R}$, y un punto interior $\boldsymbol{a}$ de $\Omega$, realizar las siguientes acciones para hallar la derivada de $f$ en $\boldsymbol{a}$.

a. Desencapsular el objeto $f^{\prime}(a)$ del esquema de derivada para verificar si existe la derivada de $f$ en $a$, y notarlo como $\mathrm{D} f(a)$,

$$
D f(a)=f^{\prime}(\boldsymbol{a})=\lim _{t \rightarrow 0} \frac{f(\boldsymbol{a}+t)-f(\boldsymbol{a})}{t} .
$$

\section{F. Derivada de una función vectorial, DIFV.}

1. Dado $\Omega \subseteq \mathbb{R}$, la función $f$ vectorial de variable real (15) y un punto interior $\boldsymbol{a}$ de $\Omega, w$

$$
\begin{aligned}
f: \Omega \subseteq \mathbb{R} & \rightarrow \mathbb{R}^{m}, m>1 \\
x & \rightarrow f(x)=\left(f_{1}(x), \cdots, f_{m}(x)\right)
\end{aligned}
$$

Para calcular la derivada $f$ en $\boldsymbol{a}$ realizar las siguientes acciones sobre los objetos.

a. Realizar la acción E1a para cada función real de valor real, $f_{1} \cdots, f_{m}$, verificando que exista

$$
f_{i}^{\prime}(a)=\lim _{t \rightarrow 0} \frac{f_{i}(a+t)-f_{i}(a)}{t}, i=1,2, \cdots, m .
$$

b. Formar el vector $\left(f_{1}^{\prime}(a), \cdots, f_{m}^{\prime}(a)\right)$, en $\mathbb{R}^{m}$, con las acciones F1a.

2. Interiorizar las acciones en procesos.

a. Realizar las acciones F1a y F1b para varios puntos interiores $a$ de $\Omega$.

b. Realizar las acciones F2a para otras funciones vectoriales de variable real $f$, cambiando la dimensión $m$ del espacio $\mathbb{R}^{m}$.

c. Considerar las acciones F2a y F2b como un proceso transforma la función vectorial de varia- 
ble real $f$ y un punto interior $\boldsymbol{a}$ de $\Omega$, en el vector, $\left(f_{1}^{\prime}(a), \cdots, f_{m}^{\prime}(a)\right)$, de $\mathbb{R}^{m}$.

3. Encapsular F2c en objeto. Considerar el proceso F2C como un elemento del espacio de las funciones vectoriales de variable real derivables en y $\Omega$ notarlo, $D f(a)=f^{\prime}(a)=\left(f_{1}^{\prime}(a), \cdots, f_{m}^{\prime}(a)\right)$ para poder realizar acciones como calcular su norma, aplicar reglas de derivación para funciones vectoriales de valor real.

\section{G. Derivada de un campo escalar, DECE.}

1. Dado un subconjunto $\Omega$ de $\mathbb{R}^{m}$, la fórmula de un campo escalar $f$ definido en (16) y un vector fijo $\boldsymbol{a}$ en el interior de $\Omega$ realizar la siguiente acción para calcular la derivada de $f$ en $a$.

$$
\begin{aligned}
f: \Omega \subseteq \mathbb{R}^{n} & \rightarrow \mathbb{R}, \quad n>1 \\
x & \rightarrow f(x)=y=f\left(x_{1}, \cdots, x_{n}\right) .
\end{aligned}
$$

a. Desencapsular el objeto gradiente de $\nabla f(a)$ y encapsularlo en el objeto $D f(a)=f(a)=\nabla f(a)$.

\section{$H$. Derivada de un campo vectorial, DECV.}

1. Dados los objetos, un subconjunto $\Omega$ de $\mathbb{R}^{m}$, la fórmula del campo vectorial $f$ dado por (17) y un vector fijo $\boldsymbol{a}$ interior a $\Omega$.

$$
\begin{array}{r}
f: \Omega \subseteq \mathbb{R}^{n} \rightarrow \mathbb{R}^{m}, \quad n>1, m>1 . \\
x \rightarrow y=f(x)=\left(y_{1}, \cdots, y_{m}\right), \quad \text { con } \\
y_{1}=f_{1}\left(x_{1}, \cdots, x_{n}\right) \\
\vdots \\
y_{m}=f_{m}\left(x_{1}, \cdots, x_{n}\right) .
\end{array}
$$

Realizar las siguientes acciones para calcular la derivada de $f$ en el vector $a$.

a. Desencapsular el objeto gradiente de un campo escalar y verificar la existencia de $\nabla f_{j}(a)$ de todos los campos escalares $f_{j}, j=1,2, \cdots, m$. Esto es equivalente a verificar la existencia de todas las derivadas del campo escalar $f_{\mathrm{j}}$ respecto a $x_{\mathrm{i}}$, evaluadas en $a$, $\frac{\partial f_{j}}{\partial x_{i}}(a)=\lim _{t \rightarrow 0} \frac{f_{j}\left(a+t e_{i}\right)-f_{j}(a)}{t}, 1 \leq i \leq n, 1 \leq j \leq m$.

b. En caso que la acción $H 1 a$, verifique la existencia de todas las derivadas parciales, formar la matriz de orden $m \times n$,definida por (18),

$$
f^{\prime}(a)=\left[\begin{array}{c}
\nabla f_{1}(a) \\
\nabla f_{2}(a) \\
\vdots \\
\nabla f_{m}(a)
\end{array}\right]=\left[\begin{array}{cccc}
\frac{\partial f_{1}(a)}{\partial x_{1}} & \frac{\partial f_{1}(a)}{\partial x_{2}} & \cdots & \frac{\partial f_{1}(a)}{\partial x_{\mathrm{n}}} \\
\frac{\partial f_{2}(a)}{\partial x_{1}} & \frac{\partial f_{2}(a)}{\partial x_{2}} & \cdots & \frac{\partial f_{2}(a)}{\partial x_{\mathrm{n}}} \\
\vdots & & & \vdots \\
\frac{\partial f_{m}(a)}{\partial x_{1}} & \frac{\partial f_{m}(a)}{\partial x_{2}} & \cdots & \frac{\partial f_{m}(a)}{\partial x_{\mathrm{n}}}
\end{array}\right]
$$

\section{Interiorizar las acciones en procesos}

a. Realizar las acciones $H 1 a$ y $H 1 b$ para varios puntos interiores $b$ de $\Omega$.

\section{b. Realizar H2a para diferentes campos vectoriales $f$.}

c. Interpretar $\mathrm{H} 2 \mathrm{a}$ y $\mathrm{H} 2 \mathrm{~b}$ en el proceso que transforma un campo vectorial $f$ y un punto interior $a$ de $\Omega$, en la matriz $m \times n$, definida en (18).

3. Encapsular $\mathrm{H} 2 \mathrm{c}$ en el objeto derivada del campo vectorial $f$ evaluado en $\boldsymbol{a}$. Interpretar la matriz (18) como un objeto el $D f(a)=f^{\prime}(a)=J(a)$ del espacio de campos vectoriales derivables en $\Omega$, sobre el cual se realizan acciones como calcular su norma, determinar la continuidad del campo vectorial $\mathrm{D} f$.

\section{Diferencial de una función en varias varia- bles. DIFV}

1. Dada la función $f$ en varias variables, definida sobre un subconjunto $\Omega$ de $\mathbb{R}^{n}$ en $\mathbb{R}^{m}, n \geq 1$, y $m \geq 1$, un punto $\boldsymbol{a}$ interior de $\Omega$, la acción de determinar si existe una transformación lineal $T_{a}$ definida de $\mathbb{R}^{n}$ en $\mathbb{R}^{m}, T_{a}: \mathbb{R}^{n} \rightarrow \mathbb{R}^{m}$, y una función en varias variables $E_{a}$ dada por (19), 


$$
\begin{aligned}
E_{a}: \Omega \subseteq \mathbb{R}^{n} & \rightarrow \mathbb{R}^{m} \\
v & \rightarrow E_{a}(v)=E(a, v),
\end{aligned}
$$

tal que,

$$
f(a+v)=f(a)+T_{a}(v)+\|v\| E(a, v) .
$$

De manera que $E(a, v) \rightarrow 0$ cuando $v \rightarrow 0$

a. Definir el vector $x=a+v$, para un vector $v$, tal que $x$ sea punto interior de $\Omega$. b. Según sean los valores de $m$ y $n$, desencapsular la función $f$, para calcular $f(x)-f(a)$.

c. Verificar que la transformación definida en (21) es lineal,

$$
\begin{aligned}
\operatorname{Df}(a)=T_{a}: \mathbb{R}^{n} & \rightarrow \mathbb{R}^{m}, n \geq 1, m \geq 1 . \\
u & \rightarrow T_{a}(u)=\operatorname{Df}(a)(u) .
\end{aligned}
$$

Según sean los valores de $m$ y $n$, desencapsular, el objeto $\mathrm{D} f(a)$, para establecer la forma como actúa (21), para los casos establecidos en (22)

$$
\begin{aligned}
& m=1, \quad n=1, \quad T_{a}(u)=D f(a) \cdot u=f^{\prime}(a) \cdot u \text {. } \\
& m>1, \quad n=1, \quad T_{a}(u)=u \cdot D f(a)=u \cdot\left(f_{1}^{\prime}(a), \cdots, f_{m}^{\prime}(a)\right) . \\
& m=1, \quad n>1, \quad T_{a}(u)=D f(a) \cdot u=\left(\frac{\partial f(a)}{\partial x_{1}}, \cdots, \frac{\partial f(a)}{\partial x_{n}}\right) \cdot\left(u_{1}, \cdots, u_{n}\right) \\
& m>1, \quad n>1, \quad T_{a}(u)=D f(a) \cdot u=\left[\begin{array}{cccc}
\frac{\partial f_{1}(a)}{\partial x_{1}} & \frac{\partial f_{1}(a)}{\partial x_{2}} & \cdots & \frac{\partial f_{1}(a)}{\partial x_{\mathrm{n}}} \\
\frac{\partial f_{2}(a)}{\partial x_{1}} & \frac{\partial f_{2}(a)}{\partial x_{2}} & \cdots & \frac{\partial f_{2}(a)}{\partial x_{\mathrm{n}}} \\
\vdots & & & \vdots \\
\frac{\partial f_{m}(a)}{\partial x_{1}} & \frac{\partial f_{m}(a)}{\partial x_{2}} & \cdots & \frac{\partial f_{m}(a)}{\partial x_{\mathrm{n}}}
\end{array}\right]\left[\begin{array}{c}
u_{1} \\
u_{2} \\
\vdots \\
u_{n}
\end{array}\right]
\end{aligned}
$$

d. Evaluar la función en varias variables $E_{a}$ definida por (23) en el punto $v=x-a$.

$$
\begin{aligned}
E_{a}: \Omega \subseteq \mathbb{R}^{n} \rightarrow \mathbb{R}^{m} & \\
\qquad & v \rightarrow E_{a}(v)=E(a, v)= \begin{cases}\frac{f(a+v)-f(a)-D f(a)(v)}{\|v\|_{n}}, & \text { si } v \neq 0 \\
0, & \text { si } v=0 .\end{cases}
\end{aligned}
$$

e. Calcular la norma del vector $v,\|v\|_{n}$.

f. Analizar el comportamiento de $E(a, v)$, calculada en $I 1 d$, cuando $\|v\|_{n}$ se aproxima a 0.

g. Si se cumple en IIf que $E(a, v) \rightarrow 0$, cuando $\|v\|_{m} \rightarrow 0$, a la transformación lineal $T_{a}=D f(a)$ definida en $/ 1 c$, denominarla diferencial de $f$ en $a$ y afirmar que $f$ es diferenciable en a.

2. Interiorizar las acciones anteriores en proceso

a. Realizar 11 para funciones $f$ cambiando valores de $m$ y $n$, y los puntos interiores de $a$ de $\Omega$. 
b. Interpretar 12 a como una proceso que determina si una función $f$ en varias variables definida en un subconjunto $\Omega$ de $\mathbb{R}^{n}$, según sea los valores de $m$ y $n$, y un punto interior $a$ de $\Omega$, si $f$ es diferenciable en $a$.

Si $f$ es diferenciable el proceso genera la transformación lineal $\mathrm{D} f(a)$, la cual transforma vectores $u$ de $\mathbb{R}^{n}$ en $\mathbb{R}^{m}$ y aproxima $f(a+v)-f(a)$, por la aplicación lineal $D f(a)(v)$ y calcula el error de esta aproximación como o $\|v\| E(a, v)$, que tiende a 0 cuando el $v$ tiende al vector nulo, 0 .

3. Encapsular el proceso 12 en el objeto, diferencial de $f$ en el punto $a$, y representarla como $D f(a)$.

\section{Conclusiones}

La DG del concepto en estudio permite diseñar un camino cognitivo para la comprensión del concepto de DFVV en un punto fijo $a$ interior del dominio de definición de la función, que significa la posibilidad de aproximar la función en vecindades de un punto por una transformación lineal. Este procedimiento requiere construir el objeto $f^{\prime}(a)$ desencapsularlo para definir la transformación lineal $\mathrm{D} f(a)$ y verificar que está aproxima a la función en puntos cercanos del punto, al analizar que el error de aproximación tiende a cero.

El análisis de la comprensión de la DFVV es complejo, requiere de varios esquemas como: la topología en $\mathbb{R}^{n}$, para los conceptos de conjunto, abierto, punto interior, vecindad; función, para analizar el tipo de función dependiendo de su dominio y recorrido; el esquema de límite, generalizándolo a funciones de varias variables; el esquema de derivada de una función; el esquema de transformación lineal que está definida por el operador derivada; el esquema de espacios vectoriales sobre los cuales se definen, normas, vectores unitarios, producto interior. Estos esquemas interactúan al definir y analizar la función error en varias variables para establecer si la función es diferenciable y si es así determinar su diferencial.
Las construcciones mentales: acción, proceso, objeto y esquema de la diferenciabilidad se pueden caracterizar al utilizar variedad de funciones, tanto definidas explícitamente como generalizadas, como por ejemplo: la constante, lineal, polinómica, racional y compuesta. En particular, es conveniente analizar funciones que presenten problemas de continuidad en algunos puntos para lograr en los estudiantes el paso de proceso a la concepción de objeto matemático, lo que les permita relacionar los conceptos de continuidad, diferenciabilidad y propiedades de las funciones diferenciables.

\section{Agradecimientos}

Este trabajo es parte del proyecto de tesis doctoral titulado: La comprensión de la diferencial del concepto de diferencial de una función en varias variables, del programa de doctorado en ciencias de la educación de la UPTC-Rudecolombia.

\section{Referencias}

Aldana, E. (2011). Comprensión de la integral definida en el marco de la teoria "APOE" (Tesis doctoral). Universidad de Salamanca, Salamanca, España.

Apóstol, T. M. (1988). Calculus. Cálculo con funciones de varias variables y álgebra lineal, con aplicaciones a las ecuaciones diferencial y a las probabilidades. Barcelona: Reverté.

Arnon, I., Cottrill, J., Dubinsky, E., Oktac, A., Roa Fuentes, S., Trigueros, M., \& Weller, K. (2014). APOS Theory, A Framework for Research and Curriculum Development in Mathematics Education. New York: Springer Science.

Artigue, M. (1986). The notion of differential for undergrate students in sciencie. Proccedings of the $X$ th annual conference of the international group for the psychology of matematic education, 229-234. 
Asiala, M. B. (1996). A Framework for Research and Development in Ungraduate Mathematics education. Research in Collegiate Mathematics Education. CBMS issues in mathematics, 6, 1- 32.

Asiala., M., Cottill, J., Dubinky, E., \& Schwingendorf, K. (1997). The development of student's graphical understanding of the derivate. JornalMathematical Beahavior 16 (4), 399-431.

Azcárate, C. (1990). La velocidad: Una introducción al concepto de derivada ( Tesisi de doctorado no publicada). Universitat Autónoma de Barcelona, Barcelona.

Baker, B., Cooley, L., \& Trigueros, M. (2000). A calculus graphing schema. The Journal for Research in Mathematics Educations, 31(5), 557 - 578.DOI: 10.2307/749887.

Bartle, R. (1975). The elements of real analysis. New York: Jhon Wiley \& Sons.

Cornu, B. (1983). Apprentissage de la notion de límite: conceptions et obstacles. Grenoble: Université I de Grenobl.

Cottrill, J. D. (1996). Understanding the limit concept: begining with a coordinated process scheme. Journal of Mathematical Behavior, 15(2), 167-192. DOI: 10.1016/S0732-3123(96)90015-2

Davis, R. B. (1986). The notion of limit: some seemingly unavoidable misconception stages. Journal of Mathematical Behaviour, 5 (3), 281-303.

Dubinsky, E. (2000). Reflective Abstraction in Advanced Mathematical Thinking. En D. Tall, Advanced Mathematical Thinking (págs. 95-123). New York: Kluwer Academic Publishers.

Dubinsky, E. W. (2005). Some historical issues and paradoxes regarding the concept of infinity: An APOS analysis: Part 1. Educational Studies in
Mathematics, 58, 335-359. DOI: 10.1007/s10649005-2531-z

Dubinsky, M., \& McDonald, M. (2001). APOS: A constructivist theory of learning in undergrad mathematics education. En H. D., The teaching and learning of mathematics at university level: An ICMI study (págs. pp. 273-280). Dordrecht: Kluwer Academic Publishers.

Galindo, F., Sanz, J., \& Tristán, L. A. (2005). Cálculo infinitesimal en varias variables. Guía Practica. Madrid: Paraninfo.

García , M., \& LLinares, S. (1996). El concepto de función através de los textos escolares: reflexión sobre una evolución. Qurrículum 10-11, 103-115.

Gómez, P.l., \& Delgado, J.R. (2012). La diferenciabilidad de funciones en varias variables, una propuesta de tratamiento metodológico. Acta latinoamericana de matemática educactiva, 25.

Gutiérrez, L., \& Valdivé, C. (2012). Una descomposición genética del concepto de derivada. Gestión y Gerencia, 6(3), 104-122. Recuperado de http://www.ucla.edu.ve/DAC/ investigacion/gyg/GyG\%202012/Diciembre\%20 2012/5-\%20LucybethGutierrez.pdf

Leinhardt, G., Zaslavsky, O., \& Stein, M. (1990). Functions, Graphs, and Graphing: Tasks, Learning, and Teaching. review of Educational Research, 60 (1), 1-64.

Martínez-Planell, R., \& Trigueros, M. (2009). Student's ideas on functions of two variables: Domain, range and representations. Proceedings of 31st annual meeting of the American Chapter of International Group for the Psychology of Mathematics Education, 5, 7-80.

Ohlsson, S. (1988). Mathematical meaning and applicational meaning in the semantics of 
fractions and related concepts. Number concepts and operations in the middle grades, 53-91.

Ohlsson, S., Ernest, A. M., \& Ress, E. (1992). The cognitive complexy of learning and doing arithmetic. Journal for Research in Mathematics Education.23(5), 441-467. DOI: 10.2307/749565.

Phillips, E.G. (1931). The teaching of differential. The Mathematical Gazette, 15, 401-403.

Piaget, J. (1972). The principles of Genetic Epistemology. (W. Mays, Trad.). London: Neubauer, P. B. (original published 1970).

Piaget, J. (1980). Psicología y pedagogía. Buenos Aires: Ariel.

Piaget, J., \& García, R. (1983). Psicogénesis e historia de la ciencia. Madrid: Siglo ventiuno editores.

Piaget, J., Choquet, G., Dieudonné, J., \& Thom, R. (1986). La enseñanza de las matemáticas modernas. Madrid: Alianza.

Roa-Fuentes, S., \& Oktac, A. (2012). Validación de una descomposición genética de transformación lineal: un análisis refinado por la aplicación del ciclo de investigación de la teoría APOE. Revista Latinoamericana de Investigación en Matemática Educativa, 15(2), 199-232. Recuperado de http:// www.redalyc.org/articulo.oa?id=33523165004

Robinson, A. (1966). Non-Standard analysis. Amsterdam, Holland. : North Holland.

Sánchez, M.T. (2012). Límite finito de una función en un punto: fenómenos que organiza (Tesis doctoral). Universidad de Granada, Granada-España.

Sánchez-Matamoros, G., Garcia, M., \& Llinares, S. (2008). La comprensión de la derivada como objeto de investigación en didáctica de la matemática. Revista Latinoamericana de investigación en Matemática Educativa. 11(2), 267-296. Recuperado de http://www.scielo.org.mx/scielo. php?pid=S1665-24362008000200005\&script $=$ sci_ arttext

Sánchez-Matamoros, G., García, M., \& Llinares, S. (2006). El desarrollo del Esquema de derivada. Enseñanza de las ciencias, 24(1), 85-98. Recuperado de http://www.raco.cat/index.php/ensenanza/ article/viewFile/73534/84742

Sierra, M.G., Gonzalez, M.T., \& Lopez, C. ( 1999). Evolución histórica del concepto de "límite funcional" en los libros de texto de bachillerato y curso de orientacion universitaria (COU): 19401995. Enseñanza de las ciencias, 17(3), 463-476. Recuperado de http://www.raco.cat/index.php/ Ensenanza/article/view/21606/21440

Stewart, J. (2002). Cálculo multivariable. Mexico: Thompson. Learning.

Tall, D. (1991). The psychology of advanced mathematical thinking. En D. Tall, Advanced mathematical thinking (págs. 3-21). Dordrecht: Kluwer.

Thomas, G., Finney, R., \&Weir, M. (1999). Cálculo con Geometría Analítica. 6a. edición. Bogotá: AddisonWesley Iberoamericana.

Trigueros, M. (2005). La noción de esquema en la investigación en matemática educativa a nivel superior. Educación matemática, 17(1), 5-31. Recuperado de http://www.redalyc.org/ pdf/405/40517101.pdf

Trigueros, M., \& Martínez-Planell, R. (2007). Visualization and abstraction: Geometric representation of functions of two variables. Proceedings of the 29th Annual Conference of the North American Chapter of the International Group for the Psychology of Mathematics Education (págs. 100-107). NV: University of Nevada, Reno: T. Lamberg \& L.R. Wiest. 